\title{
Limits on spin-dependent WIMP-proton cross-sections from the neutrino experiment at the Baksan Underground Scintillator Telescope
}

\author{
Olga Suvorova* \\ Institute for Nuclear Research of Russian Academy of Sciences, \\ prospect 60-th October 7A, Moscow 117312, Russia \\ E-mail: suvorova@cpc.inr.ac.ru

\section{Musabi Boliev} \\ Institute for Nuclear Research of Russian Academy of Sciences, \\ Baksan Neutrino Observatory, Kabardino-Balkariya 400900, Russia \\ E-mail: boliev2005eyandex.ru
}

\section{Sergei Demidov}

Institute for Nuclear Research of Russian Academy of Sciences, prospect 60-th October 7A, Moscow 117312, Russia

E-mail: demidov@ms2.inr.ac.ru

\section{Stanislav Mikheyev ${ }^{\dagger}$}

Institute for Nuclear Research of Russian Academy of Sciences, prospect 60-th October 7A, Moscow 117312, Russia

E-mail: mikheyev@pcbai10.inr.ruhep.ru

We present updated results of the Baksan Underground Scintillator Telescope in search for a signal from dark matter particles annihilating in the center of the Sun. Based on the performance of the Baksan telescope measuring upward through-going muons with energy larger $1 \mathrm{GeV}$, we confirm the absence of observable excess in arrival directions towards the Sun relatively the expectations from neutrinos of atmospheric origin for 24.12 years of live time. We conclude that the $90 \%$ C.L. upper limits on elastic scattering cross sections of dark matter WIMP (Weakly Interactive Massive Particle) off proton are comparable with correspondent limits of other operating neutrino telescopes. The best value of the limit is $3 \times 10^{-4}$ picobarn in spin-dependent (SD) interaction of WIMP on proton for mass range about $100 \div 200 \mathrm{GeV}$.

VIII International Workshop on the Dark Side of the Universe,

June 10-15, 2012

Rio de Janeiro, Brazil

\footnotetext{
*Speaker.

${ }^{\dagger}$ We dedicate this paper to the memory of our colleague S.P.Mikheyev, who died on 2011 April 23. From the begining of operation of the Baksan Underground Scintillator Telescope till recently Stas was a leader of the neutrino experiment and he is greatly missed. His carrier spanned widely of neutrino investigations including famous MSW matter effect in a theory of neutrino oscillations.
} 


\section{Introduction}

Neutrino channel in multiwave searches for relic dark matter (DM) particles is accessible within data analysis of neutrino telescopes in their regular observations of local sources like the Sun where DM could be gravitationally trapped and further accumulated for the time of solar system age. Up-to now there are no hints on excess of neutrino events in the direction towards the Sun as compared with expected background of atmospheric neutrinos at all neutrino telescopes. However, these observations allow to set the upper limits on properties of dark matter particles, in particular, the limit on the cross sections of elastic scattering of WIMP on nucleons. Due to the Sun chemical composition with approximately $73 \%$ of hydrogen, the most sensitive quantity is the spin dependent cross sections of dark matter WIMP on proton as it can be seen from the latest results of SuperKamiokande [1] and IceCube [2] collaborations.

The sensitivity of neutrino telescopes strongly depends on their muon energy threshold $E_{\mu}^{t h}$, especially in probing of light masses of WIMP. The Baksan Underground Scintillator Telescope [3] (further referred as the Baksan or the BUST) has muon energy threshold around $1 \mathrm{GeV}$, allowing for searches for neutrino signal from annihilation of relatively light WIMPs in the Sun. Minimal mass for WIMPs which could be probed at the Baksan is about $10 \mathrm{GeV}$. This is intrinsic challenge in light of recent claimed spectral peculiarities and their interpretations by many authors as an evidence on existence of light relic WIMPs both in low background detectors of nuclear recoil reactions (see review by J.Cooley at this conference) and in cosmic ray experiments of $\gamma, e^{-} e^{+}$and $p \bar{p}$ measurements (see review by A.Morselli at this conference).

Here we present the preliminary results of the Baksan experiment with the statistics twice as compared to earlier results [4], [5] and with a new analysis improved in several ways. Firstly, the previous Baksan results [4], [5] were obtained in the framework of a specific model - MSSM (Minimal Supersymmetric Standard Model) with neutralino as a dark matter candidate (see review of N.Fornengo). Performing the scan over its parameter space but fixing the mass of dark matter particle, there have obtained conservative upper limits on muon flux and the rate of dark matter annihilation in the Sun. Now rather then studying a specific model we follow present day tendency and consider specific dark matter annihilation channels, namely annihilations into $b \bar{b}, \tau^{+} \tau^{-}$and $W^{+} W^{-}$. This was also done because the previous tactics makes it difficult to compare the Baksan limits with the results of other experiments. Second, we take into account current knowledge about neutrino properties in solving the transport of oscillating and interacting neutrinos produced in the center of the Sun with energy larger few GeV.

\section{Experiment, triggers and sample of upward through-going muons}

The Baksan Underground Scintillator Telescope is 34-years continuously operating cosmic rays detector with $4 \pi$-geometry for arrival penetrating charged particles. Main parameters of the telescope have been presented in details elsewhere [3], [4]. Separation of arrival directions between up and down hemispheres is made by time-of-flight method with a time resolution $5 \mathrm{~ns}$ [3]. It was shown [3] that $95 \%$ of $1 / \beta$ values (ratios of speed of light and measured particle track velocity) lie in range of 0.7-1.3 ns for single downward going muons. Such interval but with negative sign is used to select upward going muons generated by neutrino interactions in down hemisphere. 
Telescope is located at the altitude $1700 \mathrm{~m}$ above see level in the Baksan valley of the North Caucause and at the depth $850 \mathrm{~g} / \mathrm{cm}^{2}$ under the mountain Andyrchi where the flux of atmospheric downgoing muons is reduced in 5000 times but it is still higher than upgoing muons flux in six orders of magnitude. Trajectories of penetrating particles are reconstructed by the positions of hit tanks, which put together a system of 3,150 liquid scintillation counters of standard type $(70 \mathrm{~cm} \times$ $70 \mathrm{~cm} \times 30 \mathrm{~cm})$ in configuration of parallelepiped $(17 \mathrm{~m} \times 17 \mathrm{~m} \times 11 \mathrm{~m})$. The counters entirely cover all its sides and two horizontal planes inside at the distances $3.6 \mathrm{~m}$ and $7.2 \mathrm{~m}$ from the bottom. Each plane is separated from another one by concrete absorber of $160 \mathrm{~g} / \mathrm{cm}^{2}$. The configuration provides $1.5^{\circ}$ of angular accuracy.

There are two hardware triggers used to select upward going muons. They reduce initial rate of downward going muons approximately by factor of $10^{3}$. Trigger I covers the zenith angle range $95^{\circ} \div 180^{\circ}$ while trigger II selects horizontal muons in the range $80^{\circ} \div 100^{\circ}$. The hardware trigger efficiency of $99 \%$ has been measured with the flux of atmospheric muons. These two triggers give about 1,800 events per day for further processing. In the year 2000 the telescope data acquisition system was upgraded and allowed to simplify the trigger system down to one general trigger with the rate of $17 \mathrm{~Hz}$. All raw information is than undergone further selection using off-line reconstruction code.

Selection criteria of neutrino events in the Baksan neutrino experiment did not changed since first data analyses [3]. Few requirements to be satisfied in preliminary selection were determined before the telescope was launched: only single trajectory of penetrating particle and a negative value of measured $\beta$. Additionally all events with negative values of $\beta$ were scanned by eyes to check possible misinterpretation. Also required to have an enter point of each trajectory lower than exit point in a range not less than a tank sizes. To reduce background from downward going atmospheric muons coming from smaller thickness of mountain but be scattered at large angles, all events with azimuths $0^{\circ}<\phi<180^{\circ}$ in a sample of trigger II are rejected. It was found 1700 upward through going muons for 24.12 years of live time (l.t.) survived these cuts among detected events since December of 1978 till November of 2009.

Next level of cuts impacts on events mimicked by downward going atmospheric muon interactions or muon groups: for the reconstructed muon trajectories its path inside the telescope had to be larger than thickness $500 \mathrm{~g} / \mathrm{cm}^{2}$ (that corresponds to muon energy threshold about $1 \mathrm{GeV}$ ) with both enter and exit points; selection only events with $-1.3<1 / \beta<-0.7 \mathrm{~ns}$; edge conditions on enter and exit for events in sample of horizontal events. Totally 1255 events for 24.12 1.t. years survived all cuts. From Fig. 1 (left) one can conclude about stable rate of measured upward through-going muons during all years of observation.

\section{The Sun survey during three decades}

In search for neutrinos from the dark matter processes in the Sun, we analyse distribution of measured events as a function of $\cos \left(\Psi_{\mu-\text { Sun }}\right)$ where $\Psi_{\mu-\text { Sun }}$ is the angle between upward incoming muons and the Sun position. In Fig. 1 (right) the obtained cosine distribution is presented. The mean rate per bin is shown by blue line. We estimate the background expected from atmospheric neutrinos directly from real data and shifted (false) Sun positions. Here we follow our previous analysis [4], [5] where it was shown that this method is compatible with Monte Carlo (MC) simu- 

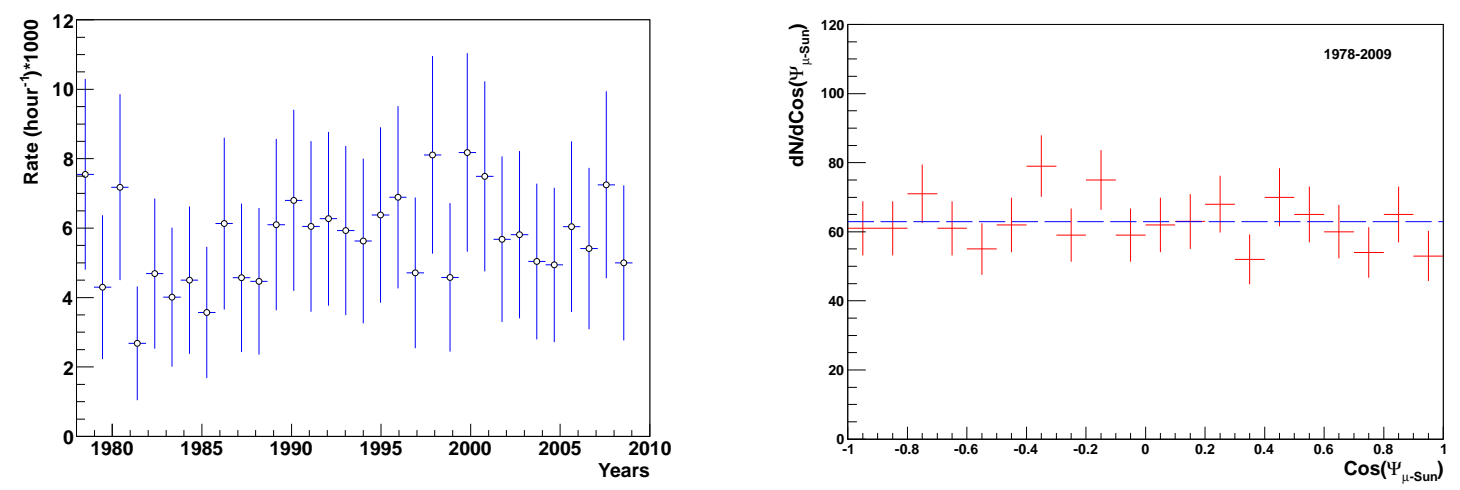

Figure 1: Left: Measured rate of upward through going muons at the BUST since December 1978. Right: Distribution of measured events in angle $\Psi_{\mu \text {-Sun }}$ between incoming neutrino events at the BUST and the Sun position. The direction of the Sun corresponds to $\cos \left(\Psi_{\mu-\operatorname{Sun}}\right)=1$.

lations of the detector response. Mean energy of simulated neutrinos, which produce muons with energy larger $1 \mathrm{GeV}$ to cross the Baksan telescope, is about $50 \mathrm{GeV}$. From comparison of data sample for 21.15 years of 1.t. [6] and MC statistics of time larger than real data taking by factor 22 (i.e., in total of 460 years) it was found that the ratio of observed total number of events to expected one without neutrino oscillations is $0.87 \pm 0.03$ (stat.) \pm 0.05 (syst.) \pm 0.15 (theor.). The details has been presented elsewhere [6].

At the location of the Baksan telescope $\left(43,16^{\circ} \mathrm{N}\right.$ and $\left.42,41^{\circ} \mathrm{E}\right)$ the Sun is seen in average about half of time per day during a year in both hemispheres; the real bin contents of solar zenith $(\theta)$ distributions reconstructed from data time information (red points with error bars) and one (blue histogram) calculated using the Positional Astronomy Library [7] are shown in Fig. 2 (left). The reconstructed values are obtained from a sample of 1255 selected upward going muons for
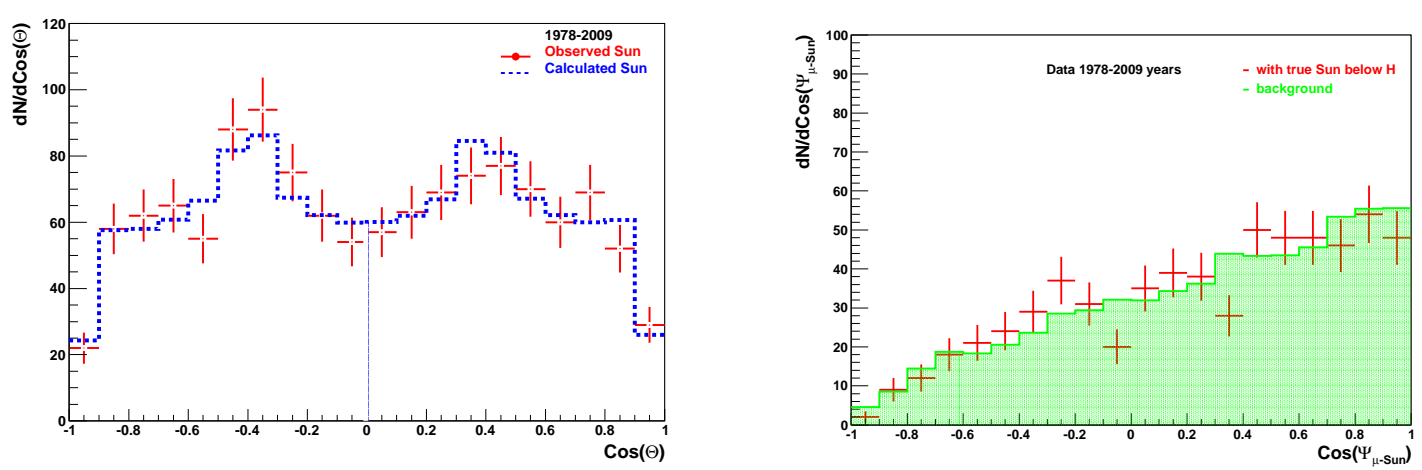

Figure 2: Left: Zenith distributions of the Sun during the BUST runtime: observed (red points with error bars) and calculated (blue, see text). Right: Distributions of $\cos \left(\Psi_{\mu-\text { Sun }}\right)$ for events toward true Sun position below horizon (red) in comparison with measured background (green) obtained from the distributions for $\cos \left(\Psi_{\mu-\text { Sun }}\right)$ averaged over six shifted Sun positions. 
24.12 years of live time. The calculated distribution is normalized to this number. As it can be seen that our multi-years measurements reproduce the Sun full-year passing track with a good accuracy. That is the base for our further analysis.

Potentially the neutrinos from dark matter annihilations in the Sun could be found at night time when the Sun is below horizon. For "night" neutrinos we compare cosine distribution in Fig. 2 (right) and integrated angular distribution in Fig. 3 with respect to measured background which has

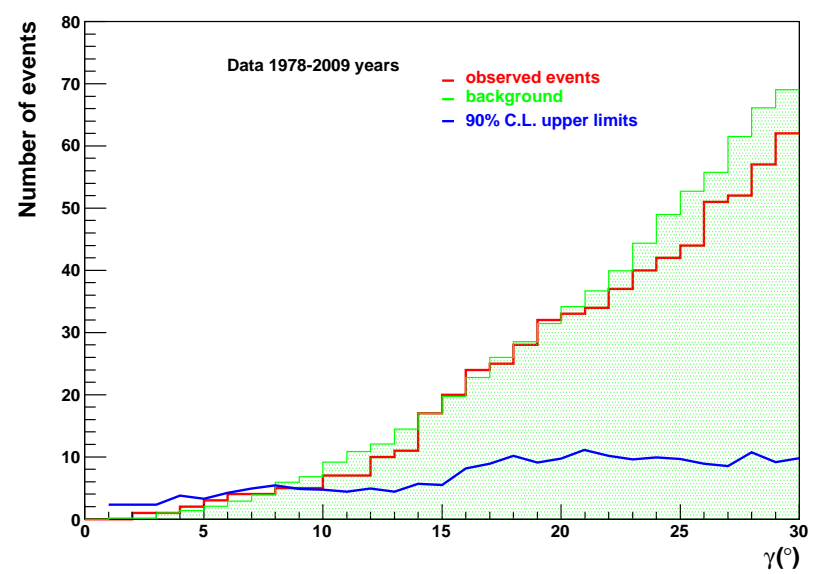

Figure 3: The 90\% C.L. upper limits on additional number of events toward the Sun (blue) in cone halfangles $\gamma$. Also shown are measured events (red) and background (green) in the same cones.

been obtained from averaged distribution of six cases of false Sun positions shifted over ecliptic. In Fig. 3 shown on abscissa value $\gamma$ is a half open angle of the cone toward the Sun. We observe no an excess of events coming from the Sun and set the $90 \%$ C.L. upper limits on additional events in each cone as also shown in Fig. 3. Assuming Poisson statistics for both expected background $\left(N_{B}\right)$ and observed events $\left(N_{o b s}\right)$ we obtain upper limits C.L. on additional number of signal events $\left(N_{S}\right)$ as follows [8]:

$$
C . L .=1-\frac{e^{-\left(N_{B}+N_{S}\right)} \sum_{0}^{N_{o b s}} \frac{\left(N_{B}+N_{S}\right)^{n}}{n !}}{e^{-N_{B}} \sum_{0}^{N_{o b s}} \frac{N_{B}{ }^{n}}{n !}} .
$$

Further we translate these results into limits on annihilation rates and muon fluxes from the Sun direction, considering in details a transport of high energy neutrinos from the Sun to the Baksan detector and found dependence on WIMP mass of value $\gamma$ where $90 \%$ of $N_{S}$ events are collected.

\section{Transport of solar neutrinos with energy higher few $\mathrm{GeV}$}

In this Section we briefly describe techniques which we used for numerical simulations of muon signal in the BUST and leave the details for future publication [9]. Resulting neutrino spectrum from WIMP annihilations is a mixture of inclusive spectra from decays of produced copious particles. Spectral characteristics of this spectrum depends on the WIMP mass and its annihilation channel. To obtain model-independent bounds we consider WIMP of masses from $10 \mathrm{GeV}$ to $1 \mathrm{TeV}$ 
with three dominant annihilation channels: $b \bar{b}, W^{+} W^{-}$and $\tau^{-} \tau^{+}$. The channel $b \bar{b}$ represents an example of "soft" spectrum while $W^{+} W^{-}$and $\tau^{+} \tau^{-}$are examples of "hard" spectra.

We use our own $\mathrm{C}$ code for MC simulation of neutrino propagation from the center of the Sun to the level of the Baksan detector. At the point of production in the Sun, we take neutrino spectra for each annihilation channel obtained in Ref. [10]. When simulating neutrino propagation we take into account oscillations in the Sun matter and in the vacuum, CC (charged current) and NC (neutral current) scattering of neutrino with interior of the Sun including $\tau$ regeneration. Our procedure of MC simulations is very similar to that of in WimpSIM package [11, 12]. Neutrino oscillations in $3 \times 3$ scheme were implemented according to the algorithm presented in Refs. [13, 14] which is very convenient for using with varying density of the Sun. For parameters of neutrino mixing matrix we use the best fit of experimental data of Ref. [15]. Below we present the results for normal hierarchy only. We use standard solar model presented in Ref. [16]. Neutrino-nucleon DIS cross sections and distributions are calculated according to formulas presented in [17] and CTEQ6 [18] parton distribution functions. Finally, we calculate flux of muons produced by neutrinos in the rock below the Baksan telescope as in Ref. [20]. We check results obtained by our code for neutrino flux at the level of the Earth with that of obtained by WimpSIM [11, 12] and find an agreement with a sufficient accuracy [9].

\section{Results and limits on neutralino-proton cross sections}

If the capture and annihilation of WIMPs in the Sun reach exact equilibrium for time much less than age of the solar system 4,5 Giga years, the annihilation rate $\Gamma_{A}$ is equal to a half of capture rate defined by WIMPs scattering cross sections off solar matter. And in this case, the limit on muon flux from dark matter annihilation in the Sun and the limit on annihilation rate can be recalculated to limit on elastic cross section of dark matter particle on nucleons [21, 22]. According to that annihilation rate can be divided into two pieces

$$
\Gamma_{A}=\Gamma_{A}\left(\sigma_{S I}\right)+\Gamma_{A}\left(\sigma_{S D}\right)
$$

where $\Gamma_{A}\left(\sigma_{S I}\right)$ and $\Gamma_{A}\left(\sigma_{S D}\right)$ are the parts of equilibrium annihilation rate determined by either spin-dependent (SD) or spin-independent (SI) interactions.

To obtain the upper limit on SD elastic cross sections we use recalculation procedure described in [22] where we obtained corresponding coefficients in the following expressions

$$
\sigma_{S D}^{U p p L i m}\left(m_{\chi}\right)=\lambda^{S D}\left(m_{\chi}\right) \cdot \Gamma_{A}^{U p p L i m}\left(m_{\chi}\right)
$$

as functions of neutralino mass $m_{\chi}$. The (preliminary) results for the limits on SD elastic cross section from Baksan's data for the annihilation channels $W^{+} W^{-}, b \bar{b}$ and $\tau^{+} \tau^{-}$are presented in Figure 4 (left) along with corresponding limits of SuperKamiokande [1] and IceCube [2]. Typically, only $W^{+} W^{-}$and $b \bar{b}$ channels are considered in the limit on dark matter annihilation rate or SD elastic cross section. We include also $\tau^{+} \tau^{-}$channel because of two reasons. Firstly, it allows to extend the limits on SD cross section for "hard" type of neutrino spectra to lower masses of dark matter. Second, it illustrates an important point: in spite of the fact that the limits on muon 

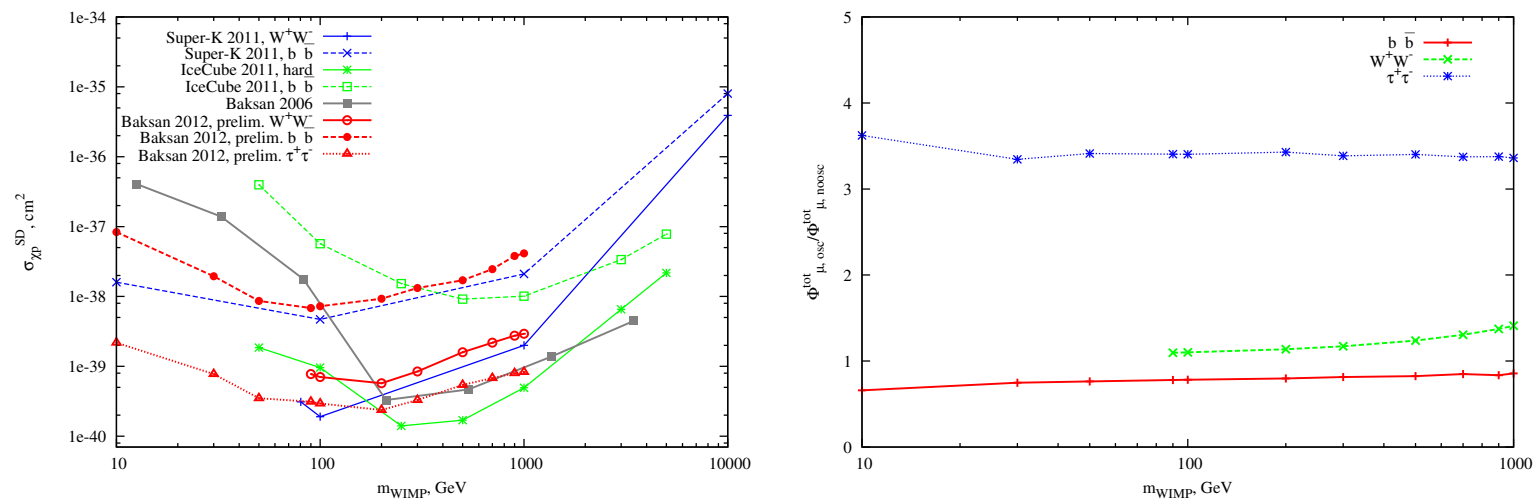

Figure 4: The Baksan limits on SD elastic cross section of WIMP on proton in comparison with other experimental results (left); ratios of expected muon fluxes both from DM neutrino and antineutrino generated in pure three annihilation branches $b \bar{b}$ quarks, or $\tau^{+} \tau^{-}$leptons or $W^{+} W^{-}$bosons in cases with and without three flavours oscillations (right).

fluxes are approximately the same for $W^{+} W^{-}$and $\tau^{+} \tau^{-}$, the limits on SD cross section (and annihilation rate) are quite different for these two channels as it can be seen in Fig. 4 (left). The explanation of this fact resides partly in the effect of neutrino oscillations: the enhancement due to this effect for $\tau^{+} \tau^{-}$channel is considerably larger than for the case of $W^{+} W^{-}$channel. This is illustrated in Figure 4 (right) where the ratio of muon fluxes with and without oscillation for different annihilation channels depending on mass of dark matter is shown. Another important effect is a breaking "democracy" in average number of high energy neutrinos per one act of dark matter annihilations: for $\tau^{+} \tau^{-}$pairs it is larger in a factor of $2 \div 3$ than for $W^{+} W^{+}$or $b \bar{b}$ channels.

It can be seen in Fig. 4 that neutrino oscillations result in decrease of muon flux from $b \bar{b}$ annihilation channel, which is expected to be dominating at lighter neutralino masses. At the same time the muon flux from $W^{+} W^{-}$annihilations is enhanced. These results allow also to interpret more correctly the previous Baksan limit [5] (brown line in Fig. 4, left) calculated without taking into account neutrino oscillations.

\section{Summary and conclusions}

We have performed updated analysis of indirect dark matter search with the Baksan Underground Scintillator Telescope data for 24.12 years of live time. Search for an excess of upward going muons in the direction toward the Sun we do not observe any significant deviation from expected atmospheric background. We have presented preliminary the 90\% C.L. upper limits on spindependent elastic cross section of dark matter on proton assuming particular annihilation channels $b \bar{b}, W^{+} W^{-}$and $\tau^{+} \tau^{-}$. These limits have been derived from the limits on muon fluxes and annihilation rates for dark matter masses in the interval $10 \div 1000 \mathrm{GeV}$. The best value of the limit is about $3 \times 10^{-4}$ picobarn for WIMP masses within $100 \div 200 \mathrm{GeV}$, that is comparable with those presented by SuperKamiokande and IceCube collaborations. 


\section{Acknowledgments}

We acknowledge our colleagues from the Baksan Observatory for permanent collaboration providing a long-term stability of the telescope readout systems. The work was supported in part by the Russian Found for Basic Research Grants 09-02-00163a and 11-02-01528a. The work of S.D. was supported by the grants of the President of the Russian Federation NS-5590.2012.2, MK2757.2012.2, by Russian Foundation for Basic Research grants 11-02-01528-a and 12-02-31726mol-a and by the Ministry of Science and Education under contract No. 8412. The numerical part of the work was performed on Calculational Cluster of the Theory Division of INR RAS.

\section{References}

[1] T. Tanaka et al. [Super-Kamiokande Collaboration], Astrophys. J. 742, 78, (2011)

[2] R. Abbasi et al. [IceCube Collaboration], Phys. Rev. D 85 (2012) 042002

[3] M.M.Boliev et al., Proc. of the 17th ICRC, Paris, France, v.7, 106, (1981).

[4] M.M.Boliev et al., Nucl. Phys., 48, 83, (1996).

[5] M.M.Boliev et al., Dark Matter in Astro- and Particle Physics 711, 1997; see also O.V.Suvorova, [arXiv:hep-ph/9911415].

[6] M.M.Boliev et al., Proc. of the First Workshop on Exotic Physics with Neutrino Telescope, Uppsala, Sweden, 19 (2006) [arXiv:astro-ph/0701333].

[7] P.T.Walance, Starlink user note 67 (1999), http://star-www.rl.ac.uk/star/docs/sun67.htx/sun67.html.

[8] Particle Data Group, Phys. Rev. D 45 (1992)

[9] S. V. Demidov, O. V. Suvorova, in preparation.

[10] M. Cirelli, N. Fornengo, T. Montaruli, I. A. Sokalski, A. Strumia and F. Vissani, Nucl. Phys. B 727 (2005) 99 [Erratum-ibid. B 790 (2008) 338] [hep-ph/0506298].

[11] M. Blennow, J. Edsjo and T. Ohlsson, JCAP 0801 (2008) 021 [arXiv:0709.3898 [hep-ph]].

[12] J. Edsjo, WimpSim Neutrino Monte Carlo, http://www.fysik.su.se/ edsjo/wimpsim/

[13] T. Ohlsson and H. Snellman, J. Math. Phys. 41 (2000) 2768 [Erratum-ibid. 42 (2001) 2345] [hep-ph/9910546].

[14] T. Ohlsson and H. Snellman, Eur. Phys. J. C 20 (2001) 507 [hep-ph/0103252].

[15] D. V. Forero, M. Tortola and J. W. F. Valle, arXiv:1205.4018 [hep-ph].

[16] J. N. Bahcall, A. M. Serenelli and S. Basu, Astrophys. J. 621 (2005) L85 [astro-ph/0412440].

[17] E. A. Paschos and J. Y. Yu, Phys. Rev. D 65 (2002) 033002 [hep-ph/0107261].

[18] J. Pumplin, D. R. Stump, J. Huston, H. L. Lai, P. M. Nadolsky and W. K. Tung, JHEP 0207 (2002) 012 [hep-ph/0201195].

[19] V. Barger, W. -Y. Keung, G. Shaughnessy and A. Tregre, Phys. Rev. D 76 (2007) 095008 [arXiv:0708.1325 [hep-ph]].

[20] A. E. Erkoca, M. H. Reno and I. Sarcevic, Phys. Rev. D 80 (2009) 043514 [arXiv:0906.4364 [hep-ph]].

[21] G. Wikstrom and J. Edsjo, JCAP 0904 (2009) 009 [arXiv:0903.2986 [astro-ph.CO]].

[22] S. Demidov and O. Suvorova, JCAP 1006 (2010) 018 [arXiv:1006.0872 [hep-ph]]. 